\title{
Application of GLBP Algorithm in the Prediction of Building Energy Consumption
}

\author{
Dinghao $\mathrm{Lv}^{1}$ \\ College of Electronic and Electrical \\ Engineering \\ Shanghai University of Engineering \\ Science \\ Songjiang District, Shanghai 201620, \\ China
}

\author{
Bocheng Zhong ${ }^{2}$ \\ College of Electronic and Electrical \\ Engineering \\ Shanghai University of Engineering \\ Science \\ Songjiang District, Shanghai 201620, \\ China
}

\author{
Jing Luo $^{3}$ \\ College of Electronic and Electrical \\ Engineering \\ Shanghai University of Engineering \\ Science \\ Songjiang District, Shanghai 201620, \\ China
}

\begin{abstract}
Using BP neural network in past to predict the energy consumption of the building resulted in some shortcomings. Aiming at these shortages, a new algorithm which combined genetic algorithm with Levenberg-Marquardt algorithm (LM algorithm) was proposed. The proposed algorithm was used to improve the neural network and predict the energy consumption of buildings. First, genetic algorithm was used to optimize the weight and threshold of Artificial Neural Network (ANN). Levenberg-Marquardt algorithm was adopted to optimize the neural network training. Then the predicting model was set up in terms of the main effecting factors of the energy consumption. Furthermore, a public building power consumption data for one month is collected by establishing a monitoring platform to train and test the model. Eventually, the simulation result proved that the proposed model was qualified to predict short-term energy consumption accurately and efficiently.
\end{abstract}

Keywords-BP Neural network; Building energy consumption; Genetic algorithm; Levenberg-Marquardt algorithm

\section{INTRODUCTION}

There are many factors affecting the energy consumption of the building, and it has the characteristics of randomness, timevarying and regionally [1]. It can be divided into three aspects: environment, structure and operation process. For a given area of buildings, the main influence factors of the building energy consumption include regional climate characteristics, buildings, residential environment, building construction and operation management of heating system [23]. Because neural network [4] has strong nonlinear, parallel processing ability and robustness, and it does not need to set up complex mathematical model, so it has been favored by the researchers. Now it is widely used in different conditions of architecture energy consumption prediction research.

The traditional algorithm exists the shortcoming of slow convergence speed and easy to fall into local minimum. Bingbing Shi [5] and others find that when using LM algorithm to improve the BP neural network, the BP network training speed is improved obviously, and the prediction error is smaller. Ahua $\mathrm{Mu}$ [6] and others by using the method of combining genetic algorithm with BP algorithm (hereinafter referred to as GABP algorithm) makes the prediction error smaller, but the amount of computation is increased and the convergence rate is slow. By the literature [7], under the same training target, the training steps of genetic neural network are 10 times of the LM algorithm, so the training speed of GABP algorithm is not ideal, and it can't satisfy the requirement of energy consumption prediction of real-time online. For this kind of situation, in this article, the GLBP algorithm which combines the GABP algorithm and the LM algorithm is adopted to establish the building energy consumption prediction model.

\section{GLBP ALGORITHM}

The improved BP neural network is mainly divided into two parts, one part is to optimize the input global variable, and to improve the quality of input variables; the other part is to optimize local variables, and to improve the convergence speed of the algorithm. Therefore, the improved BP neural network (GLBP) has two stages in the calculation: 1) Using the genetic algorithm to determine the approximate optimal approximate solution; 2) The LM algorithm is used to search the approximate solution, until you find the local optimal solution. The specific steps are as follows:

A. Genetic algorithm global optimization [8]

1) According to the network structure, to determine the code length of genetic algorithm. Using real coding, changing the parameter set $X$ and fields into the string structure space $S$,

2) The fitness function is the only standard to measure the quality of the individual. In order to improve the prediction accuracy, the reciprocal of the error sum of squares of the LM neural network is used as the fitness function. The function is:

$$
f\left(X_{i}\right)=\frac{1}{\sum_{i=1}^{k}\left(t_{i}-y_{i}\right)^{2}}
$$

Among them, $\mathrm{k}$ is the number of training samples; $\mathrm{t}$ is target output; $y$ is the actual output;

3) Determine the genetic strategy, including selected population size $n$, method of selection, crossover and mutation, and crossover probability $P_{e}$ and mutation probability $P_{m}$;

4) Population initialization, the individual of the population is encoded to calculate the initial fitness valu $f(x)$;

\footnotetext{
This work was supported by the Shanghai board of education discipline construction under Grant no. XKCZ1212, and also was supported by the Innovation Program of Shanghai University of Engineering Science under Grant No.E1-0903-14-010340
} 
5) Selection of the genetic operation method based on step 3 ), the genetic operations of selection, crossover and mutation are carried out to form the next generation population;

6) To determine whether the new individual population termination condition, if the condition is satisfied, the search will be stopped, otherwise, return to step 5), until satisfy the termination condition.

\section{B. LM algorithm local optimization [9]}

1) Selecting the largest fitness from the group which meet the conditions as the initial weights and thresholds of the training of the LM neural network;

2) Again using the training samples of LM neural network. Input samples, and then calculate, and get a response in the output layer;

3) In accordance with the direction of reducing the actual output and target output error to modify the weights of each neuron. Unlike the BP algorithm, LM algorithm uses two order derivative approximation, and the rate of convergence is much faster than BP algorithm. The LM algorithm allows the error along the direction of increasing development, not easy to fall into local minimum.

4) To determine whether the training results meet the certain indicators, not meet return 3), else end.

\section{BUILDING ENERGY CONSUMPTION PREDICTION MODEL}

Through the analysis of the factors affecting energy consumption of a particular building, considering the human activity is relatively fixed, the equipment operation and the enclosure structure basically remain unchanged all the year, so here mainly consider the influence of the outdoor climate conditions (weather) to energy consumption. According to the characteristics of the particular building energy consumption, selecting temperature, humidity and wind speed as the input layer factors, and selecting the electric energy consumption as the output layer factor. When you want to predict the future energy consumption, you just need the future weather forecast information. Here, the number of input layer neurons is three. The number of output layer neurons is one and the number of the hidden layer also is one. The energy consumption prediction model is built based on this, then the model diagram (three-ten-one) is shown in Fig one.

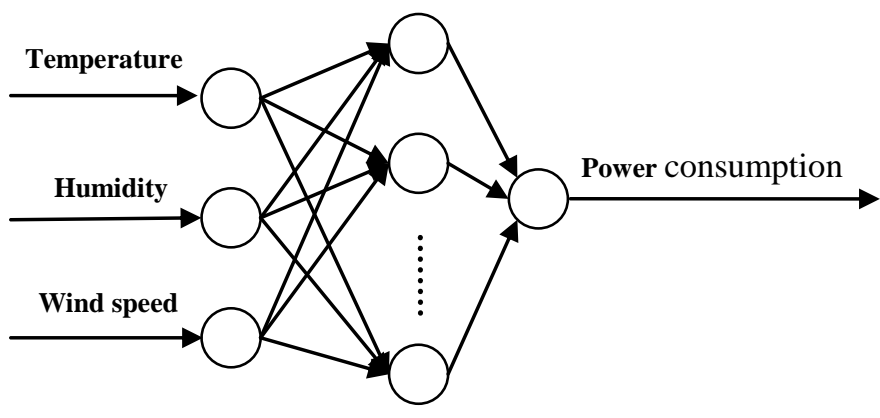

Fig. 1. Energy consumption prediction model
To obtain the experimental data, a university training center is taken as the research object, and then set up the experimental platform. Because of the large proportion of energy consumption, and other forms of energy consumption can be collected by similar methods, the energy consumption is only selected as the collection object.

Energy consumption data acquisition system uses three-tier design: the field monitoring layer, the network communication layer and management layer. The management layer sends out data acquisition command, then transfers the command to each electric meter by network communication layer. Electric meter receives the instruction and checks it, then submits the corresponding energy consumption information to management computer, and finally stores it to the database. The prediction model can obtain the training sample from the database.

\section{Simulation AND ANALYSIS}

From the database to select three months (ninety days) of raw data. Dividing these sample data into two parts. The first part is as the training sample of neural network and this part includes the data of 93 days. The remaining data is as the test samples. In order to improve the tolerance of the network, add "noise" in each group of training samples, therefore, the number of the training sample is 186.

According to the characteristics of the neural network processing data, the Premnmx function [10] is used to control the training samples normalized between $[-1,1]$, and the method is as follows:

$p^{\prime}=2 \frac{p-p_{\min }}{p_{\max }-p_{\min }}-1$

Type: $p^{\prime}$ is the normalized variable; $p$ is the primitive variable; $p_{\max } 、 p_{\min }$ respectively is the maximum and the minimum of the original variables.

Contrast the GABP algorithm with the GLBP algorithm in the simulation experiment, structural parameters of the model are consistent. Parameters of genetic algorithm: population size is $n=50$; genetic algebra is $g e n=100$; crossover rate is $P_{c}=0.5$; the mutation rate is $P_{m}=0.01$. Part of neural networks: vector is 0.3 ; the largest number of iterations is 8000 and the minimum error of the training target is 0.001 . The neuron transfer function of the network interface layer adopts s-shaped tangent function, tansig[11], the neuron transfer function of output layer adopts linear function, purelin[12].

Type: The GABP algorithm is an algorithm which combine neural network and genetic algorithm. The GLBP algorithm is proposed in this paper.

MATLAB as the simulation tool, then respectively use the GABP and GLBP algorithm to build energy consumption prediction model. After the simulation experiments, the training error curve of the test results are as follows: 


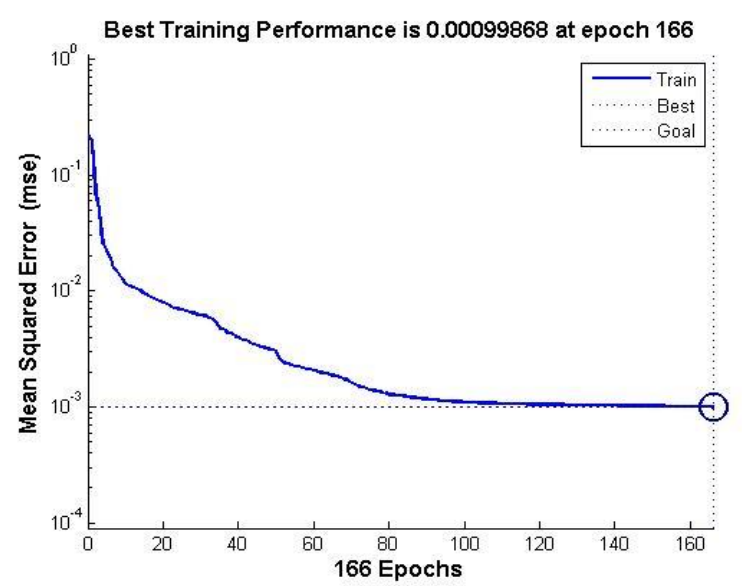

Fig. 2. GLBP training error trend chart

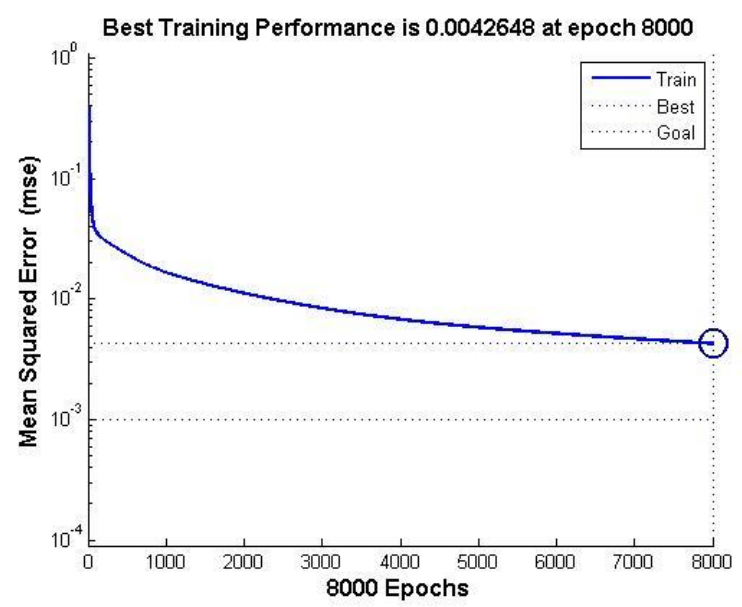

Fig. 3. GABP training error trend chart

The experimental results show that, GLBP algorithm for network training is faster than GABP algorithm, and the number of basic iterative can achieve the training objectives within 200 times, as shown in Fig 2. All of them use the same

method which uses genetic algorithm to search the optimal weights and threshold value. However, not only does the GABP algorithm increase the amount of calculation and consume a long time, but also can't make neural network jump out of the local minimum point, and it can be seen from Fig 3 . Even if the number of training reach the maximum $(8000$ times), network still failed to reach the training target. LM algorithm was used to make up the time of the searching process of genetic algorithm, and increased the speed of network training, finally it can quickly reach the goal of the training. This shows that in this paper the GLBP algorithm can satisfy the requirement of the real-time prediction of energy consumption.

Not only does the Building energy consumption prediction need the speed, but also need to meet a certain precision, to suit the requirements of engineering application. Using the GLBP to predict the energy consumption of the next 7 days, the prediction result is shown in table 1 . Overall, the maximum relative error is $3.86 \%$; the average relative error is $1.37 \%$; the prediction accuracy is relatively high, so it could meet the needs of the actual demand the building energy consumption. The forecast result of GLBP is as follows:

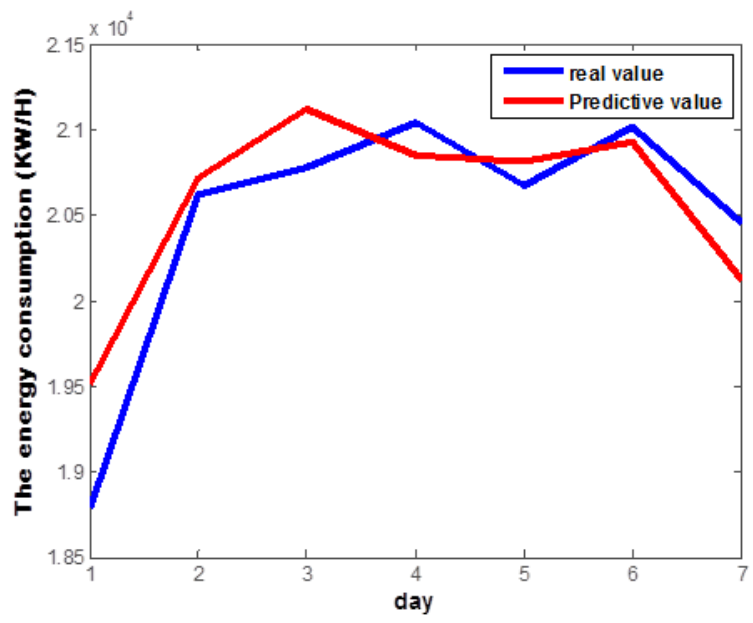

Fig. 4. The forecast results of GLBP

TABLE I. GLBP PREDICTION RESUlts

\begin{tabular}{cccccccc}
\hline date & $\mathbf{1}$ & $\mathbf{2}$ & $\mathbf{3}$ & $\mathbf{4}$ & $\mathbf{5}$ & $\mathbf{6}$ & $\mathbf{7}$ \\
\hline real value & 18787.0 & 20616.5 & 20778.5 & 21040.5 & 20673.0 & 21012.5 & 20450.5 \\
Predictive value & 19515 & 20716 & 21117 & 20850 & 20815 & 20923 & 20119 \\
relative error & $3.86 \%$ & $0.48 \%$ & $1.63 \%$ & $0.91 \%$ & $0.67 \%$ & $0.42 \%$ & $1.61 \%$ \\
\hline
\end{tabular}

\section{CONCLUSION}

According to the requirement of real-time prediction of building energy consumption in short term, this paper proposes genetic algorithm combined with the LM algorithm, namely GLBP algorithm, and this method improves the performance of neural network to predict the building energy consumption. Through the analysis of main influence factors and setting the network parameter, the building energy consumption prediction model is established based on GLBP. In order to verify the feasibility of the model, energy monitoring platform is built to collect the electric energy data, by recording the weather conditions to train and test the model, in order to improve the fault tolerance of the network, join the interference data in the training samples. The results show that the GLBP algorithm training time is short, not easy to fall into local minima, strong generalization ability, and the prediction accuracy can satisfy the requirement of the engineering application.

In the future, not only does it need to predict energy consumption in a short period of time, but also need to predict the energy consumption of a month or even a year. Long-term energy consumption prediction will have a brighter future. 


\section{REFERENCES}

[1] Zhao, Hai-xiang, and Frédéric Magoulès. "A review on the prediction of building energy consumption." Renewable and Sustainable Energy Reviews 16.6 (2012): 3586-3592.

[2] Mena, R., et al. "A prediction model based on neural networks for the energy consumption of a bioclimatic building." Energy and Buildings 82 (2014): 142-155.

[3] Binshou Tian. Building energy efficiency testing technology (second edition) [M]. Beijing: China building industry press, 2010.8.

[4] Ekici B B, Aksoy U T. Prediction of building energy consumption by using artificial neural networks [J]. Advances in Engineering Software, 2009, 40(5): 356-362.

[5] Bingbing Shi, Zhemin Duan, Zhengjun Lu. Comparative study on the neural network to predict medium and long-term power load [J]. Relay, 2007, 35(23): 43-45.

[6] Ahua Mu, Shaolei Zhou, Zhiqing Liu, etc. By using the genetic algorithm improved BP learning algorithm [J]. The computer simulation, 2005, 22(2): 150-151.
[7] Neto A H, Fiorelli F A S. Comparison between detailed model simulation and artificial neural network for forecasting building energy consumption [J]. Energy and Buildings, 2008, 40(12): 2169-2176.

[8] Alsayegh O, Almatar O, Fairouz F, et al. Prediction of the long-term electric power demand under the influence of $\mathrm{A} / \mathrm{C}$ systems [J]. Proceedings of the Institution of Mechanical Engineers, Part A: Journal of Power and Energy, 2007, 221(1): 67-75.

[9] Chen Haiying, Guoqiao, Xuli. Hundred meters based on hybrid genetic neural network prediction method [J]. The computer simulation, 2004, 21(2):89-91.

[10] Li Minqiang, Kou Jisong, Lindan, etc. The basic theory of genetic algorithms and application [M]. Beijing: science press, 2002.3.

[11] Zhao Qingyan. Genetic optimization neural network network traffic prediction [J]. Microelectronics and computer, 2013, 30 (3):133-135.

[12] Kůrková V. Kolmogorov's theorem and multilayer neural networks [J]. Neural networks, 1992, 5(3): 501-506. 